\title{
Towards a modular suturing catheter for minimally invasive vascular surgery
}

\author{
Estevan H. Murai ${ }^{1}$, Shervanthi Homer-Vanniasinkam ${ }^{2}$, Pierre G. Silveira ${ }^{3}$, Jian S. Dai ${ }^{4}$, \\ Daniel Martins $^{1}$, Helge A. Wurdemann ${ }^{6}$
}

\begin{abstract}
Endovascular aneurysm repair (EVAR) is a minimally invasive approach for abdominal aortic aneurysm (AAA) treatment. Compared to open surgery, the benefits of EVAR include faster recovery and shorter time in hospital as well as no general anesthesia (in most cases). Though EVAR has become a preferred way to treat AAA with an increasing number of procedures, there are persisting complications, e.g. stent graft migration. Suturing the stent graft to the aorta increases the displacement force necessary to move the implant. This paper describes the design of a suturing catheter for EVAR. The suturing device consist of two modules which can be inserted through the femoral arteries into the abdominal aorta where both join using an electro-magnetic connector. The positioning module provides an anchor inside the aorta for the suturing module and new sequential positions for each stitch. Our largescale prototype is validated inside a phantom vessel made of silicone material. We are able to successfully prove the concept of this novel single-sided suturing catheter for EVAR.
\end{abstract}

\section{INTRODUCTION}

Abdominal aortic aneurysm (AAA) is a disease of the human's main blood vessel that runs from the heart down through the chest and abdominal area. Risk factors such as smoking, high blood pressure, and other heart or blood vessel diseases are associated with a weakening of the aortic wall and with AAA formation [1]. The vessel will bulge which can get bigger over time and might burst (rupture), causing life-threatening bleeding. In 2013, AAA resulted in 168,200 deaths [1]. Depending on the severity, the aorta will eventually need to be repaired either through open surgery or Minimally Invasive Surgery (MIS) with the use of an endovascular stent graft [2]. Since the mid-80s, Endovascular Aneurysm Repair (EVAR) has become the preferred minimally invasive AAA treatment due to advantages over open surgery including less blood loss, shorter surgery time and reduced mortality within the first 30 days [3]. However, patients that undergo an EVAR procedure are subject to long-term complications, such as stent-graft migration [4], [5]. A stent graft migration can lead to loss of fixation and

\footnotetext{
${ }^{1}$ E. H. Murai and D. Martins are with the Mechanical Engineering Department, Federal University of Santa Catarina, Florianpolis, Brazil. eng.estevan.murai@gmail.com

${ }^{2} \mathrm{~S}$. Homer-Vanniasinkam is with the Department of Mechanical Engineering, University College London, United Kingdom and the Leeds Vascular Institute, Leeds General Infirmary, United Kingdom.

${ }^{3}$ P. G. Silveira is with the Surgery Department, Federal University of Santa Catarina, Florianpolis, Brazil.

${ }^{4}$ J. S. Dai is with the Department of Informatics, King's College London, United Kingdom.

${ }^{6} \mathrm{H}$. A. Wurdemann is with the Department of Mechanical Engineering, University College London, United Kingdom. h.wurdemanneucl.ac.uk
}
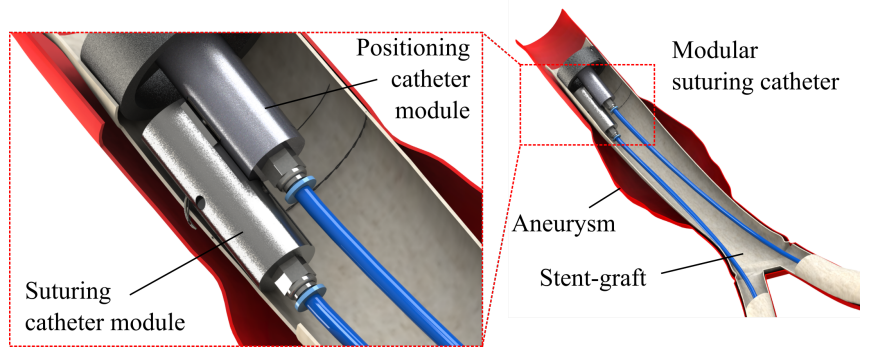

Fig. 1. 3D CAD model of the modular suturing system composed of a positioning and suturing catheter module. Each module can be inserted through the femoral arteries into the abdominal aorta where the device assembles.

leaks (called Type I endoleaks) that occur around the top stent graft. Due the blood flow into the sac caused by Type I endoleaks, the pressure inside the sac raises increasing the risk of rupture [4], [6]. Migration occurs in between $3-28 \%$ of EVAR cases in the first three years after EVAR depending on the type of stent graft [2], [7]. Studies show that reinterventions are required for up to $24 \%$ of patients due to migration in the long run [8]. In [9], researchers investigated the proximal fixation of a number of aortic stent grafts in comparison to a hand-sewn anastomosis (conducted in open surgery). They concluded that a sutured anastomosis fixates a graft better than any stent design tested.

Medical suturing devices are commercially available. However, these instruments are suture passers, hand held apparatuses that enable the surgeon to access the surgical site through a smaller incision. The surgeon needs to pass an integrated needle through the soft tissue and then feed the needle back in order to make a stitch. Hence, access to both sides of the soft tissue is necessary. A suture passer usually performs interrupted stitches or running stitches. Examples of commercial suture passers are Smith \& Nephew ${ }^{\circledR}$ FIRSTPASS ${ }^{\text {TM }}$, EndoEvolution ${ }^{\circledR}$ Endo360 30 TM Ethicon ${ }^{\circledR}$ Suture assistant ${ }^{\mathrm{TM}}$, Boston Scientific ${ }^{\circledR}$ Capio $^{\circledR}$, Apollo $^{\circledR}{ }^{\circledR}$ OverStitch ${ }^{\mathrm{TM}}$, GORE ${ }^{\circledR}$ Suture Passer ${ }^{\mathrm{TM}}$, Cayenne Medical ${ }^{\circledR}$ CrossFix $^{\circledR}$ and Quattro ${ }^{\circledR}$, Ceterix Orthopaedics ${ }^{\circledR}$ NovoStitch ${ }^{\circledR}$, Covidien ${ }^{\circledR}$ Endo Stitch $^{\mathrm{TM}}$ and Covidien ${ }^{\circledR}$ SILS ${ }^{\mathrm{TM}}$ Stitch Articulating Suturing Device. None of these commercially available endoscopic suturing devices are capable of automatically perform continuous chain stitches or lock stitches as required to fix a stent-graft to the internal aortic wall [10].

A number of researchers have investigated solutions for the endoscopic suturing problem. One approach involves 
robotizing aforementioned manual, commercially available suture passers such as the Endo360 ${ }^{\circ \mathrm{TM}}$ [11], [12], the Endo Stitch $^{\mathrm{TM}}$ device [13] or SILS ${ }^{\mathrm{TM}}$ by Covidien ${ }^{\circledR}$ [14]. The earlier mentioned challenges have not been overcome by the automation of these instruments. Another method has been proposed by designing a grasping mechanism with a high number of Degrees of Freedom (DoFs) in order to manipulate a curved needle [15], [16], [17], [18], [19], [20], [21]. Good visual feedback of the operating area is required which is not provided during EVAR procedures. In [22], [23], a suturing device is described called Heartflo ${ }^{\mathrm{TM}}$. This anastomotic device automates the suturing process simultaneously delivering 10 sutures through the graft and vessel wall - hence, a continuous suturing procedure cannot be achieved. Studies have shown that a continuous suture pattern in end-to-end anastomosis might result in the purse-string effect crimping the artery wall at high blood pressures [24], [25]. However, it is reported that the continuous pattern is considered a haemostatic suturing technique resulting in bleeding to stop immediately [26]. This sealing ability is desirable for the prevention of Type-I endoleaks. Hence, there is a need of a new automated suturing device that allows fixation of stent-grafts to the internal aortic wall using a continuous suturing pattern.

In this paper, we present a new design of a suturing catheter for EVAR allowing single-sided suturing in a minimally invasive way. The device has great potential to operate in vivo, inside the aortic root, and deliver a continuous suturing pattern around the artery circumference (see Figure 1). The suturing catheter builds on our previous work in [27] creating new kinematic structures for a single, one-sided stitching device. However, our proposed suturing device in the paper is modular and consists of a suturing and positioning module, due to size requirements. Each module can be inserted through the femoral arteries and assembled within the abdominal aorta. Here, we describe the robotic mechanism of a large-scale suturing catheter module to demonstrate its feasibility.

In Section II, procedural steps of an EVAR treatment including the proposed suturing concept is explained. Further, the requirements for a modular suturing device are summarized. Section III describes the overall suturing catheter consisting on a positioning and suturing module. In particular, the focus of this section is on the suturing mechanism. The experimental setup inside a phantom testbed and results are presented in Section IV. Section V lists the achievements of this work and identifies future steps towards a miniaturized prototype.

\section{DEVELOPING A SUTURING DEVICE FOR EVAR}

\section{A. EVAR procedure and suturing concept}

During EVAR, a catheter is inserted into an artery in the groin (called the femoral artery). The catheter is threaded up into the abdominal aorta, and the stent graft is released from the catheter. The deployment catheter is removed and the two modules of our proposed modular suturing device is inserted through each femoral artery. Inside the abdominal aorta, the suturing and positioning catheter modules will join. After suturing has been performed around the full circumference inside the aorta, both catheters will be disconnected and retrieved through the arteries in the groin.

\section{B. Device requirements}

To address the clinical needs, the suturing catheter will need to comply with a number of quantitative requirements:

- The connecting mechanism between the catheter modules should be easily detachable for timely extraction from the aorta and femoral arteries.

- A suture should keep together the stent-graft and aortic wall, and withstand pull-out forces that are at least as large as the pull-out forces of commercially available self-fixed stent-grafts [28].

- The suturing technique should allow a continuous suture pattern to prevent Type-I endoleaks.

- The clinician should be able to conduct the suturing procedure without any visual feedback from outside the patient's body.

Allowing the blood circulation to continue during the EVAR procedure (apart from the short durations when the deployment balloon is inflated within the aorta) would be an additional advantage of a suturing device. Developing medical devices that are inserted through the femoral artery must have a maximum diameter of $7 \mathrm{~mm}$, i.e. the diameter of each catheter module should be within this limitation. In this paper, we present a large-scale prototype with a diameter of $30 \mathrm{~mm}$ to proof the concept and feasibility.

\section{OVERALL SYSTEM DESIGN}

\section{A. Intra-vascular suturing through joining a positioning and suturing module}

The proposed suturing device consists of two modules/parts as shown in Figure 2: a suturing and positioning catheter module. Each module is mounted onto a catheter tip

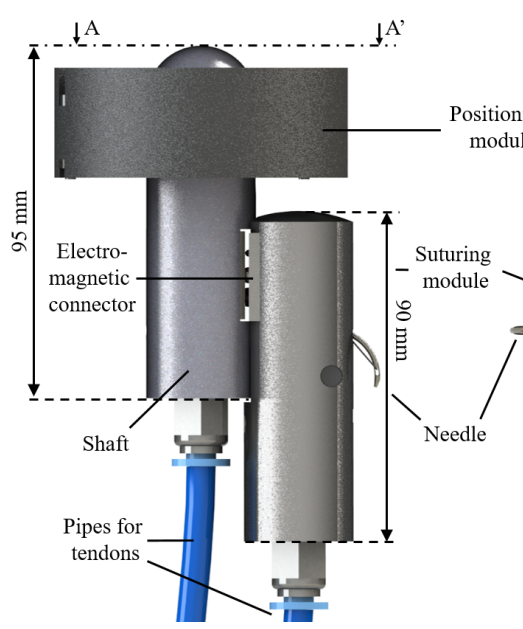

(a)

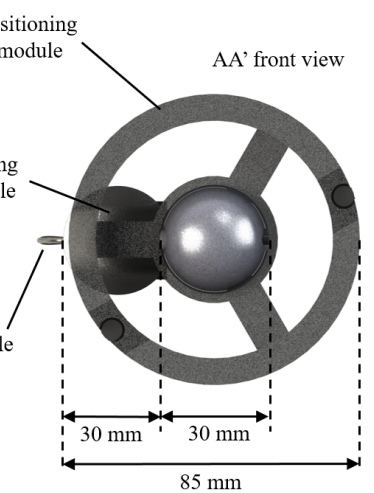

(b)
Fig. 2. (a) Top and (b) front view of the suturing catheter made of a suturing and positioning module. 


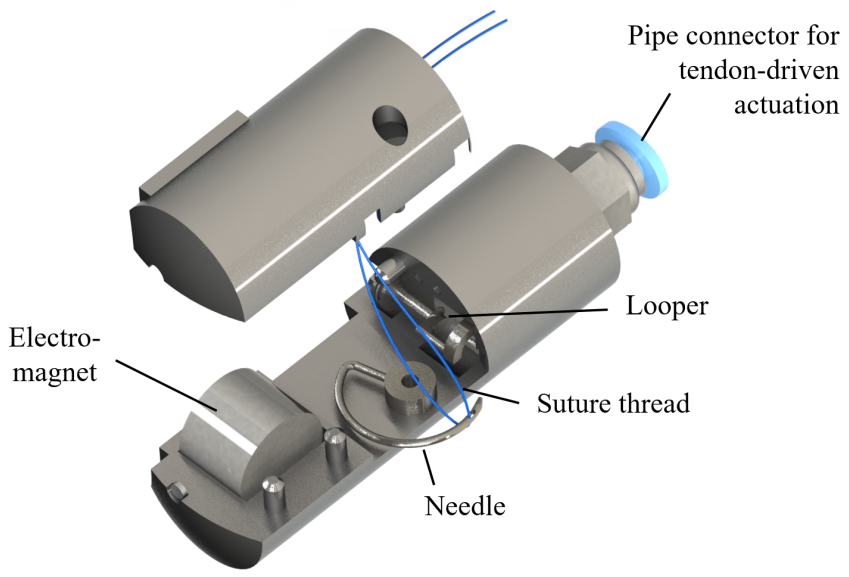

Fig. 3. Exploded view of the suturing catheter module composed of a needle, looper and an electromagnet to connect to the positioning module.

and inserted through the femoral arteries on each side of the groin into the abdominal aorta. The large-scale prototype of the positioning module has a length of $95 \mathrm{~mm}$ serving two purposes: (i) The tip acts as an anchor to the aortic wall and has a partly hollow, cylindrical structure (diameter: $85 \mathrm{~mm}$ ) allowing blood flow during the suturing procedure (as shown in Figure 2(b)). (ii) The shaft of the positioning module (diameter: $30 \mathrm{~mm}$ ) is equipped with an embedded actuated rotary joint with a small platform made of ferromagnetic material on one side (see Figure 2). The suturing catheter module connects to this platform via an electromagnetic component integrated inside the suturing module. During the suturing process, the positioning catheter provides a stable location for the suturing catheter module allowing

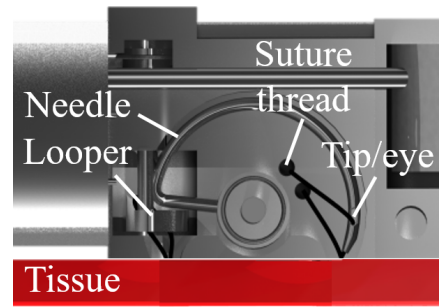

(a)

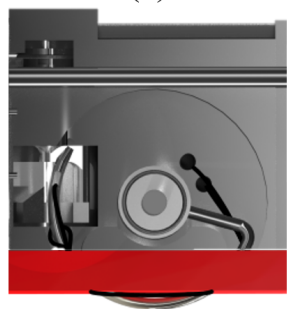

(c)

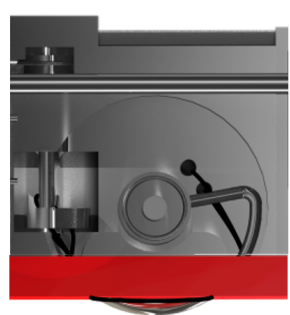

(b)

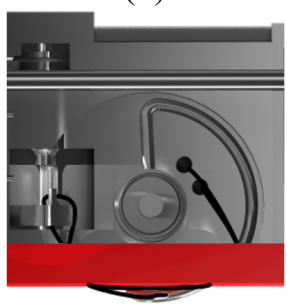

(d)
Fig. 4. The sequence of a single stitch performed by the suturing module: (a) Suture thread loop is fed through the looper and eye of needle when needle is in the default position; (b) The needle is rotated by $180^{\circ}$ until the tip feeds the thread into the looper; (c) The looper creates a loop; (d) The needle is retrieved from the looper through the new loop until it reaches the default position. a full rotation around the shaft of the positioning module. This prototype is made of Polylactic Acid (PLA) using an Ultimaker 2. Hence, the tip (anchor) of the positioning module is entirely rigid. It is envisaged to replace this part with a soft inflatable robot to allow size reductions to the diameter of the module's shaft when inserting and retrieving this catheter module. This paper focuses, in particular, on the mechanism of the suturing catheter module which is explained in the following section.

\section{B. Design of the suturing module}

As mentioned earlier, the suturing catheter module builds on our previous work in [27] creating new kinematic structures for a single, one-sided stitching device for industrial and medical applications. An exploded view of this module is illustrated in Figure 3. The prototype has a length of $90 \mathrm{~mm}$ and diameter of $30 \mathrm{~mm}$. An embedded electromagnet (Uxcell XRN-XP DC 12 V $2.5 \mathrm{kgf}$ ) is integrated into the tip connecting the suturing module to the ferromagnetic platform of the positioning module. The needle has a semi-circular shape rotating via its center point. The eye of the needle is located at the tip of the needle feeding the suture thread into the looper. The looper has an arc shape rotating around an axis passing through the arc center point. Protrusions on the looper arc secure the suture thread and keep the loop open so the needle passes inside the loop. The suturing module is made of Selective Laser Sintering (SLS) fabricated by a FORMIGA P 110, an industrial 3D printer by EOS.

The suturing module follows a four-step process to perform one stitch: The default set-up of the module is shown in Figure 4(a) where the needle is inside the device and a suture thread is fed through the eye and forms an initial loop through the looper. The needle then rotates through the tissue until a reaches the looper where a new loop is created. During the final step, the needle returns to the default position.

\section{Robotic actuation system coordinating suturing sequence}

The suturing and positioning module execute a coordinated procedure to locate each stitch by the suturing module side by side to feed the needle through the loop of the previous step and, hence, deliver a continuous suturing pattern around the artery circumference. The process is as follows: The suturing module performs a stitch creating a loop with the suture thread passed through a loop that was created by the previous stitch as explained in Section IIIB. After a single stitch is executed, the positioning catheter module moves the suturing catheter module to the next stitch location where the entire process is repeated.

These sequential steps are implemented into a robotic actuation system and interfaced with the catheter prototype as shown in Figure 5. Two antagonistic tendons are connected to each moving part: the needle, looper and rotary shaft. When a tendon pair is actuated, the respective moving part rotates (anti-)clockwise. The tendons pass from the catheter modules through two separate pipes to three unbranded MG996R $13 \mathrm{kgf} . \mathrm{cm}$ (at $4.8 \mathrm{VDC}$ ) servomotors (The motors for the needle and looper mechanisms are labeled M1 and 


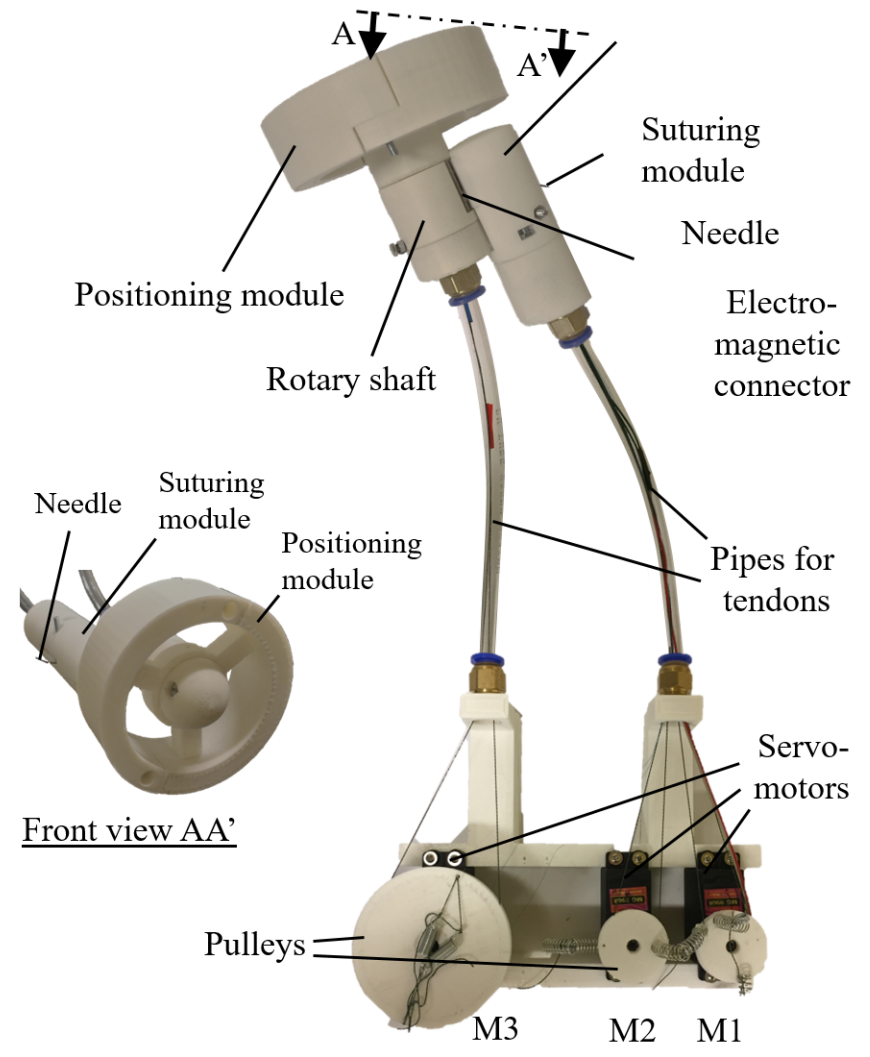

Fig. 5. Top and front (small figure) view of the large-scale prototype of the modular suturing catheter: The rotary shaft and suturing mechanism are tendon-driven and integrated in a pulley system actuated by servomotors.

M2, respectively, the motor for the positioning module M3). Each servomotor is equipped with a pulley to which the tendons are fixed to. The pulley diameters for the needle and looper are $20 \mathrm{~mm}$ and $54 \mathrm{~mm}$ for the rotary shaft of

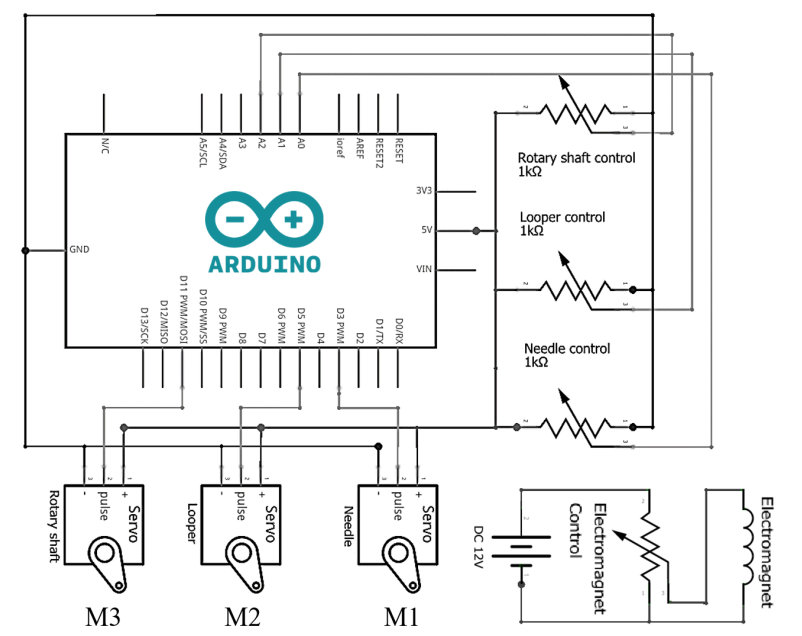

(a)

(b)

Fig. 6. (a) Control platform schematic composed of an Arduino Uno R3 development board, three servomotors and potentiometers. (b) The electromagnet force is controlled by a potentiometer connected to a power supply. the positioning module. The electromagnet of the suturing module is connected to an Aim-TTi CPX400s power supply providing $12 \mathrm{VDC}$.

The actuation platform is controlled by an Arduino Uno R3. Three $1 \mathrm{k} \Omega$ linear potentiometers connected to the development board are used to adjust the servomotors' position. Figure 6 illustrates the setup of the Arduino development board and the electronic components. The electromagnet force is controlled directly by the power supply (see Figure 6(b)). This tendon-driven robotic mechanism is located outside the patient's body and ensures the required flexibility when each module is passed through each femoral artery to the suturing location inside the aorta.

To perform a series of stitches, the motion of the suturing and positioning module are coordinated. The integrated actuation sequence is described by the following procedural steps (see Algorithm 1):

1) Actuate M1 clockwise: The needle advances from its default position through the artery wall into the looper.

2) Actuate $M 2$ anticlockwise: The looper releases the previously created loop.

3) Actuate M2 clockwise: The looper fetches the suture thread and creates a new loop.

4) Actuate M1 anticlockwise: The needle is retrieved from the looper and artery wall and moved into the default position.

5) Actuate M3 clockwise: The suture catheter module repositions the suture module to the next stitch location.

\section{EXPERIMENTAL RESULTS}

\section{A. Experimental setup inside a phantom environment}

The large-scale suturing catheter shown in Figure 5 is experimentally validated inside a large-scale aorta phantom environment. The aortic vessel for the testbed has

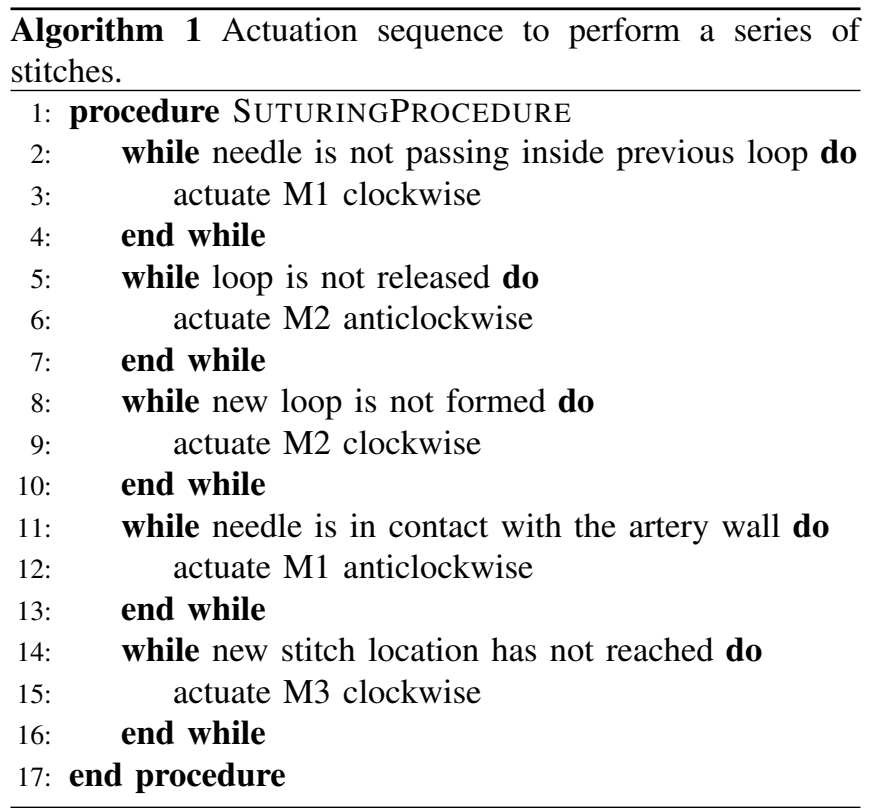




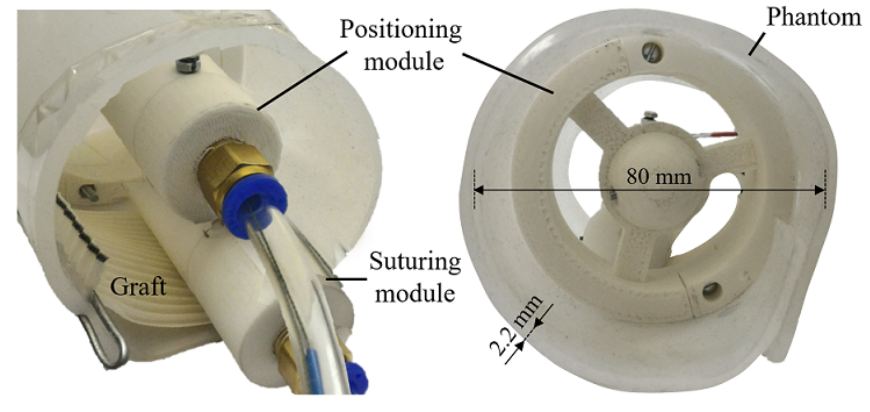

Fig. 7. Experimental setup with the suturing catheter inside a phantom vessel made of Ecoflex ${ }^{\circledR} 00-30$ silicone. The positioning module is anchored to the phantom and joined to the suturing module so that the graft sample can be sutured to the phantom vessel.

a cylindrical shape with a diameter of $80 \mathrm{~mm}$, length of $150 \mathrm{~mm}$ and thickness of $2.2 \mathrm{~mm}$. The phantom was made of Ecoflex ${ }^{\circledR}$ 00-30 silicone (Smooth-On, Inc., Easton, PA), a material that has been used in literature to mimic the aorta tissue in testbeds [29]. The suturing device is inserted into the phantom vessel. The setup is presented in Figure 7. All experiments are performed with a $5 / 0$ monofilament polypropylene suture thread. During the suture procedures, a sample of a Uni-Graft ${ }^{\circledR}$ K DV by B. Braun was used to be sutured inside the phantom vessel.

\section{B. Experimental results}

After the suturing catheter performed a number of stitches, the phantom vessel was cut along the longitudinal axis to assess the suture pattern. Figure 8 shows the experimental results. The catheter performed five stitches along $30 \mathrm{~mm}$ of the vessel circumference. Each stitch has been successfully executed creating a sequence of intersecting loops (as

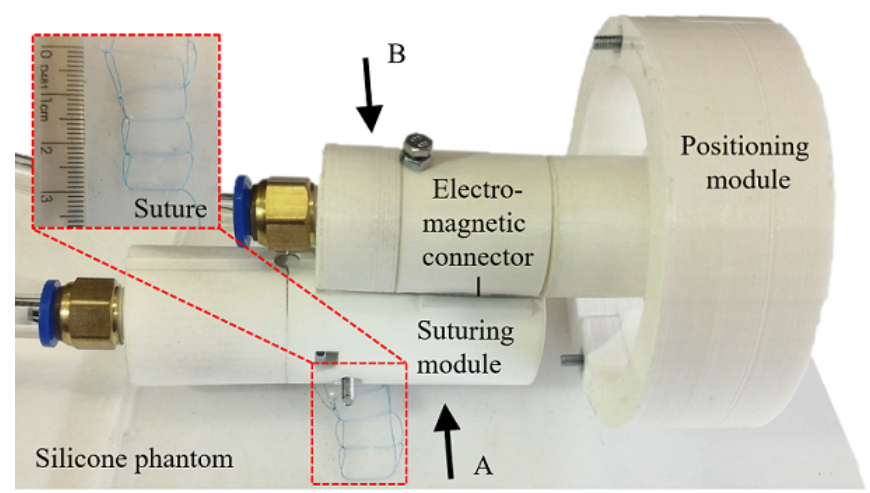

(a)

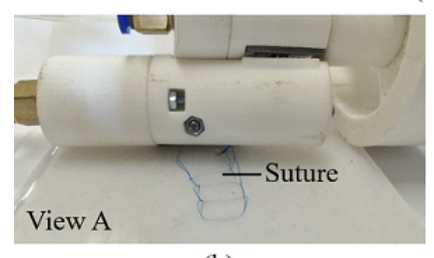

(b)

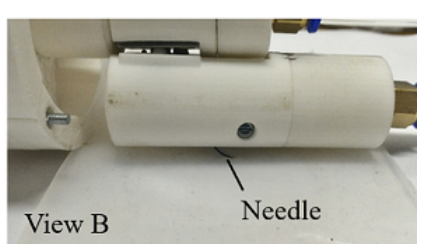

(c)
Fig. 8. (a) Side view of the suturing catheter with the open phantom vessel. Close-up side views show (b) the successful suture and (c) the insertion of the needle into the phantom. described in Section III-C). Using an electronic scale, the strength of the suture was measured. The suture was able to withstand forces of $17.45 \mathrm{~N}$ before the suture ruptured.

The experiments showed that the large-scale suturing catheter is able to successfully perform a suture pattern inside a phantom vessel. The positioning module provides stability for the suturing module. Even with a few stitches along a short length, the suture strength performs better than a number of self-fixed stent-grafts [28].

\section{CONCLUSIONS}

This paper has presented the novel design, development and testing of a new suturing catheter for EVAR allowing single-sided suturing in a minimally invasive way. The device consists of two modules which can be introduced separately through each femoral artery into the abdominal aorta. Here, both catheters join via an electro-magnetic connector. The positioning module provides an anchor and rotary guidance for the suturing module. A new kinematic suturing structure allows for a single, one-sided stitching procedure. We demonstrated the feasibility of a large-scale suturing prototype inside a phantom environment successfully performing sutures which are able to withstand forces of $17.45 \mathrm{~N}$.

As mentioned in Section II-B, strict dimensional requirements are defined for medical devices aiming to be introduced through femoral arteries for in vivo use. Hence, we aim to reduce this large-scale prototype so that the final diameter of the catheter is within $7 \mathrm{~mm}$. This includes the creation of a soft robotic mechanism/structure for the positioning module (similar to the devices in [30], [31], [32], [33]) that can be crimped or folded when introducing the catheter into the femoral artery and, then, deployed inside the aorta. So, by controlling the pressure inside the soft robotic catheter, the size can be adapted to different aorta diameters.

During experimental suturing procedures, two failure modes have been identified: (i) the looper releases the suture thread before a complete loop has been generated and (ii) the looper does not release the suture thread. Future work could explore to embed sensors (similarly to [34], [35]) into the suturing module to coordinate the sequential steps in a more reliable way. In addition, a number of experiments will be conducted to validate the suture with respect to its ability to prevent Type-I endoleaks, dislocation and pull-out forces [28].

\section{ACKNOWLEDGEMENT}

The work presented in this paper was supported by the International Cooperation Program Special Visiting Researcher $n^{\circ} 9 / 2014-1^{\circ}$ schedule financed by CAPES, Brazilian Federal Agency for Support and Evaluation of Graduate Education within the Ministry of Education of Brazil. The authors would also like to thank University College London and King's College London for the support.

\section{REFERENCES}

[1] GBD 2015 Mortality and Causes of Death Collaborators, Global, regional, and national life expectancy, all-cause mortality, and causespecific mortality for 249 causes of death, 1980-2015: a systematic analysis for the Global Burden of Disease Study 2015, Lancet, vol. 388(10053), pp. 1459-1544. 
[2] E.J. Waasdorp, J.-P.P.M. de Vries, A. Sterkenburg, J.-A. Vos, H.J.C. Kelder, F.L. Moll, C.K. Zarins, The association between iliac fixation and proximal stent-graft migration during EVAR follow-up: Mid-term results of 154 talent devices, Eur. J. Vasc. Endovasc. Surg., vol. 37(6), pp. 681-687, 2009.

[3] F.A. Lederle, J.A. Freischlag, T.C. Kyriakides, F.T. Padberg, J.S. Matsumura, T.R. Kohler, P.H. Lin, J.M. Jean-Claude, D.F. Cikrit, K.M. Swanson, P.N. Peduzzi, Open Versus Endovascular Repair (OVER) Veterans Affairs Cooperative Study Group FT. Outcomes following endovascular vs open repair of Abdominal Aortic Aneurysm: A Randomized Trial, J. Am. Med. Assoc., vol. 302(14), pp.1535-1542, 2009.

[4] The United Kingdom EVAR Trial Investigators, Endovascular versus open repair of Abdominal Aortic Aneurysm, N. Engl. J. Med., vol. 362(20), pp. 1863-1871, 2010.

[5] M. Sugimoto, A. Koyama, K. Niimi, A. Kodama, H. Banno, K. Komori, Long-term comparison of endovascular and open repair of Abdominal Aortic Aneurysms: Retrospective analysis of matched cohorts with propensity score, Vasc. Endovasc. Surg., in press, 2017.

[6] M.A. Heikkinen, J.M. Alsac, F.R. Arko, R. Metsanoja, A. Zvaigzne, C.K. Zarins, The importance of iliac fixation in prevention of stent graft migration, J. Vasc. Surg., vol. 43(6), pp.1130-1137, 2006.

[7] K. Spanos, C. Karathanos, V. Saleptsis, A.D. Giannoukas, Systematic review and meta-analysis of migration after endovascular Abdominal Aortic Aneurysm repair, Vascular, vol. 24(3), pp.323-336, 2016.

[8] A. Kaladji, E. Steintmetz, Y. Goueffic, M. Bartoli, A. Cardon, Long-Term Results of Large stent grafts to treat Abdominal Aortic Aneurysms, Ann. Vasc. Surg., vol. 29(7), pp. 1416-1425, 2015.

[9] T. Resch, M. Malina, B. Lindblad, J. Malina, J. Brunkwall, K. Ivancev, The impact of stent design on proximal stent-graft fixation in the abdominal aorta: an experimental study, Eur. J. Vasc. Endovasc. Surg., vol.20(2), pp. 190-195, 2000

[10] P.D.H. Lokuge, Design of remote endoscopic suturing device, Ph.D. thesis, Dept. Mech. Eng., Massachusetts Institute of Technology, 2003.

[11] S. Leonard, K.L. Wu, Y. Kim, A. Krieger, P.C. Kim, Smart tissue anastomosis robot (STAR): A vision-guided robotics system for laparoscopic suturing. IEEE Trans. Biomed. Eng., vol. 61(4), pp. 13051317, 2014.

[12] S. Leonard, A. Shademan, Y. Kim, A. Krieger, P.C. Kim, Smart Tissue Anastomosis Robot (STAR): Accuracy evaluation for supervisory suturing using near-infrared fluorescent markers. IEEE Int. Conf. Robot. Autom., pp. 1889-1894, 2014.

[13] T. Goepel, F. Haertl, A. Schneider, M. Buss, H. Feussner, Automation of a suturing device for minimally invasive surgery. Surg. Endosc., vol. 25(7), pp. 2100-2104, 2011.

[14] T. Looi, B. Yeung, M. Umasthan, J. Drake, KidsArmAn image-guided pediatric anastomosis robot. IEEE/RSJ Int. Conf. on IROS, pp. 41054110, 2013.

[15] A. Kapoor, N. Simaan, R.H. Taylor, Suturing in confined spaces: constrained motion control of a hybrid 8-DoF robot, IEEE Int. Conf. on Advanced Robotics, pp. 452-459, 2005.

[16] Y. Kurose, Y.M. Baek, Y. Kamei, S. Tanaka, K. Harada, S. Sora, A. Morita, N. Sugita, M. Mitsuishi, Preliminary study of needle tracking in a microsurgical robotic system for automated operations, Int. Conf. on Control, Automation and Systems, pp. 627-630, 2013.

[17] R.C. Jackson, R. Yuan, D.-L. Chow, W.S. Newman, M.C. Çavuşoğglu, Real-Time Visual Tracking of Dynamic Surgical Suture Threads, IEEE Trans. Autom. Sci. Eng., vol. PP(99), pp.1-13, 2017.

[18] S. Iyer, T. Looi, J. Drake, A single arm, single camera system for automated suturing, IEEE Int. Conf. Robot. Autom., pp. 239-244, 2013.

[19] S. Sen, A. Garg, D.V. Gealy, S. McKinley, Y. Jen, K. Goldberg, Automating multi-throw multilateral surgical suturing with a mechanical needle guide and sequential convex optimization, IEEE Int. Conf. Robot. Autom., pp. 4178-4185, 2016.

[20] C. Staub, T. Osa, A. Knoll, R. Bauernschmitt, Automation of tissue piercing using circular needles and vision guidance for computer aided laparoscopic surgery, IEEE Int. Conf. Robot. Autom., pp. 4585-4590, 2010.

[21] H. Wang, S Wang, J. Ding, H. Luo, Suturing and tying knots assisted by a surgical robot system in laryngeal MIS. Robotica, vol. 28(2), pp. 241-252, 2010.

[22] S. Martens, M. Dietrich, M. Doss, A. Moritz, G. Wimmer-Greinecker, The heartflo device for distal coronary anastomoses: clinical experi- ences in 60 patients, Ann. Thorac. Surg., vol. 74(4), pp. 1139-1143, 2002.

[23] S. Martens, M. Doss, A. Moritz, G. Wimmer-Greinecker, Sutured Coronary Artery Grafting Utilizing the Heartflo Anastomosis DeviceFirst Clinical Experiences, J. Thorac. Cardiovasc. Surg., vol. 50(1), pp. 1-4, 2002

[24] N. Baumgartner, P.B. Dobrin, M. Morasch, Q.S. Dong, R. Mrkvicka, Influence of suture technique and suture material selection on the mechanics of end-to-end and end-to-side anastomoses. J. Thorac. Cardiovasc. Surg., vol. 111(5), pp. 1063-1072, 1996.

[25] P. Tozzi, D. Hayoz, P. Ruchat, A. F. Corno, C. Oedman, U. Botta, L.K. von Segesser, Animal model to compare the effects of suture technique on cross-sectional compliance on end-to-side anastomoses, Eur. J. Cardiothorac Surg., vol. 19(4), pp. 477-481, 2001.

[26] A. Cuschieri, G. Hanna, Essential surgical practice: higher surgical training in general surgery. CRC Press, 2015.

[27] E.H. Murai, H. Simas, D. Martins, New kinematic structures for oneside stitching devices, $\mathrm{ABCM}$ Int. Congress of Mechanical Engineering, 2015, doi://10.20906/CPS/COB-2015-1664.

[28] N. Melas, T. Perdikides, A. Saratzis, N. Saratzis, D. Kiskinis, D.H Deaton, Helical EndoStaples enhance endograft fixation in an experimental model using human cadaveric aortas, J. Vasc. Surg., vol. 55(6), pp. 1726-1733, 2012.

[29] T. Yamamoto, B. Vagvolgyi, K. Balaji, L.L. Whitcomb, A.M. Okamura, Tissue property estimation and graphical display for teleoperated robot-assisted surgery. IEEE Int. Conf. Robot. Autom., pp. 4239-4245, 2009.

[30] A. Stilli, H.A. Wurdemann, K. Althoefer, Shrinkable, stiffnesscontrollable soft manipulator based on a bio-inspired antagonistic actuation principle. IEEE/RSJ Int. Conf. on Int. Robots and Systems pp. 2476-2481, 2014

[31] F. Maghooa, A. Stilli, H.A. Wurdemann, K. Althoefer, Tendon and pressure actuation for a bio-inspired manipulator based on an antagonistic principle. IEEE Int. Conf. on Robotics and Automation, pp. 2556-2561, 2015

[32] H.A. Wurdemann, A. Stilli, K. Althoefer, An antagonistic actuation technique for simultaneous stiffness and position control. Int. Conf on Intelligent Robotics and Applications, pp. 164-174, 2015.

[33] A. Stilli, H.A. Wurdemann, K. Althoefer, A Novel Concept for Safe, Stiffness-Controllable Robot Links. Soft Robotics, vol. 4(1), pp. 1622, 2016.

[34] H.A. Wurdemann, S. Sareh, A. Shafti, Y. Noh, A. Faragasso, D.S Chathuranga, H. Liu, S. Hirai, K. Althoefer, Embedded electroconductive yarn for shape sensing of soft robotic manipulators. in Int. Conf. of the IEEE Engineering in Medicine and Biology Society, pp. 8026-8029, 2015.

[35] Y. Noh, H. Liu, S. Sareh, D. Suresh Chathuranga Katudampe Vithanage, H.A. Wurdemann, K. Rhode, K. Althoefer, Image-based Optical Miniaturized Three-Axis Force Sensor for Cardiac Catheterization. IEEE Sensors Journal, vol. 16(22), pp. 7924-7932, 2016. 